\title{
Réflexions relatives au traitement des tendinopathies par infiltration de PRP
}

\author{
Reflexions about the treatment of tendinopathies by PRP injection

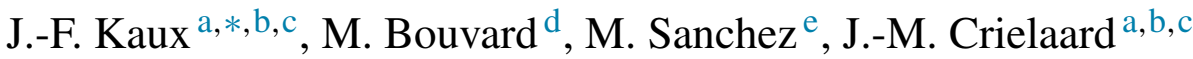 \\ a Service de médecine physique et traumatologie du sport, CHU de Liège, avenue de l'Hôpital, B35, 4000 Liège, Belgique \\ ${ }^{\mathrm{b}}$ Service pluridisciplinaire de médecine et traumatologie du sport (SPORTS2), CHU de Liège, avenue de l'Hôpital, B35, 4000 Liège, Belgique \\ ${ }^{\mathrm{c}}$ Département des sciences de la motricité, université de Liège, allée des Sports, B21, 4000 Liège, Belgique \\ ${ }^{\mathrm{d}}$ Centre de biologie et médecine du sport, 4, boulevard Hauterive, 64000 Pau, France \\ e Unidad de Cirugía Artroscópica, Beato Tomás de Zumarraga 10, 01008, Vitoria-Gasteiz, Espagne
}

Disponible sur Internet le 1 février 2015

\section{Résumé}

Introduction. - L'infiltration de plasma riche en plaquettes (PRP) représente une thérapeutique récente pour les tendinopathies chroniques. Cependant, ce traitement demeure controversé en fonction des études cliniques.

Synthèse des faits et résultats. - En se basant sur la littérature et notre expérience, nous proposons quelques pistes afin d'améliorer ce traitement. L'optimisation de la technique d'obtention du PRP est primordiale. Les différents facteurs de risques doivent être corrigés et les bonnes indications soigneusement sélectionnées. Enfin, une rééducation post-infiltration demeure absolument nécessaire.

Conclusion. - Une standardisation de l'emploi du PRP demeure nécessaire afin d'en optimiser les résultats.

(C) 2015 Elsevier Masson SAS. Tous droits réservés.

Mots clés : Plasma riche en plaquettes ; PRP ; Rééducation ; Optimisation

\section{Abstract}

Introduction. - Platelet rich plasma injection (PRP) represents a recent therapy for chronic tendinopathies. However, this treatment remains controversial in clinical studies.

Summary of the facts and results. - Based on literature and our experience, we offer few tracks in order to improve this treatment. The optimization of the technique of obtaining the PRP is paramount. Different risk factors must be corrected and the good indications carefully selected. Finally, a post-infiltration rehabilitation remains absolutely necessary.

Conclusion. - Standardisation of the use of the PRP remains necessary in order to optimize the results.

(C) 2015 Elsevier Masson SAS. All rights reserved.

Keywords: Platelet rich plasma; PRP; Rehabilitation; Optimization

\section{Introduction}

L'infiltration de plasma riche en plaquettes (PRP) représente une thérapeutique récente dans la prise en charge des tendinopathies chroniques. Cependant, ce traitement reste toujours controversé $[1,2]$; les différentes techniques de préparation du

\footnotetext{
* Auteur correspondant.

Adresse e-mail : jfkaux@chu.ulg.ac.be (J.-F. Kaux).
}

PRP et la variété des protocoles thérapeutiques rendent difficile la comparaison des études. En se basant sur la littérature et notre expérience, nous proposons quelques pistes afin d'optimiser ce traitement.

\section{Préparation du PRP}

La variabilité de résultats pourrait s'expliquer par une confusion concernant les «PRP»[3]. Selon leur protocole 
de préparation, les «PRP» présentent des concentrations plaquettaires, érythrocytaires et leucocytaires très variables. Une optimisation de la technique de récolte-concentration et une standardisation de l'injection demeurent nécessaires. La concentration plaquettaire devrait idéalement atteindre 3 à 4 fois celle du sang complet, à savoir entre 600000 et 900000 plaquettes par micro-litre [4-6]; une concentration supérieure à 1200000 plaquettes pourrait effectivement s'avérer néfaste [6-8]. On devrait pouvoir injecter un PRP reproductible (concentration plaquettaire identique) chez tous les patients [9]. La présence de cellules des lignées blanche ou rouge s'avère délétère pour le processus de cicatrisation tissulaire, liée à une réaction inflammatoire plus importante, néfaste au processus cicatriciel (dégradation de la matrice extra-cellulaire, inhibition de la synthèse de collagène) [6,10-12]. De plus, il a été démontré que l'effet anti-bactérien du PRP contre les Staphylococcus aureus, Staphylococcus epidermidis, Propionibacterium acnes et Staphylococcus aureus méticillino-résistants (MRSA) n'était pas lié à la présence de GB [13]. Actuellement, contrairement à toutes les autres techniques de préparation du PRP, seule la collecte plaquettaire via une machine d'aphérèse permet d'atteindre aisément ces objectifs [9].

\section{Correction des facteurs de risques des tendinopathies}

Avant d'envisager toute infiltration de PRP, un diagnostic précis étayé et une recherche et correction d'éventuels facteurs métaboliques (diabète, hyperuricémie, hypercholestérolémie, dysthroïdie) sont indispensables [14]. L'efficacité du PRP pourrait être compromise par la prise médicamenteuse de quinolones, corticoïdes (per os ou en infiltration) ou statines [14]. Ces familles médicamenteuses accélèrent la dégénérescence tendineuse pouvant éventuellement entraîner leur rupture $[15,16]$.

\section{Indications des infiltrations de PRP}

Les patients susceptibles de bénéficier d'infiltrations de PRP devront être sélectionnés : les indications pertinentes (mais toujours discutées dans la littérature) concernent les tendinopathies corporéales chroniques (patellaires, calcanéennes), rebelles aux traitements conservateurs (y compris une rééducation excentrique prolongée et les ondes de choc), sans conflit osseux ni luxation [17]. Les conflits osseux (maladie de Haglund, conflit sous-acromial avec un acromion Bigliani de type III, séquelles de maladie de Sinding-Larsen-Johansson...) devront impérativement faire l'objet d'une correction chirurgicale avant d'envisager une éventuelle infiltration adjuvante par PRP. Les enthésopathies (patellaires proximales, calcanéennes, épicondylites) pourraient théoriquement bénéficier d'une infiltration PRP, même si les résultats demeurent souvent moins favorables, tout comme ceux d'autres traitements (ex : ondes de choc), en raison de leur structure histologique particulière. Concernant les pathologies inflammatoires (ex: enthésopathie dans le cadre d'une spondylarthropathie), il est indispensable de traiter la pathologie inflammatoire sous-jacente.

\section{Protocole thérapeutique}

Même si le produit diffuse à partir du site de l'injection [18], il conviendrait que celle-ci se réalise sous contrôle échographique, tout en respectant les précautions d'asepsie [19]. Une anesthésie locale est déconseillée sous peine de compromettre le potentiel thérapeutique du PRP; l'anesthésie pourrait effectivement réduire le $\mathrm{pH}$ local, responsable d'une inhibition et d'une diminution, voire absence de dégranulation plaquettaire [20]. Même si le PRP s'active naturellement au contact du collagène dénaturé, une activation préalable $\left(\mathrm{ex}: \mathrm{CaCl}_{2}\right)$ permettrait une dégranulation plaquettaire et une libération plus rapide de facteurs de croissance, effectivement impliquées lors de la cicatrisation tissulaire [5].

Les suites post-infiltratives, régulièrement douloureuses, peuvent justifier d'une cryothérapie locale et de la prise d'antalgiques. Les AINS seront évités, au-moins de j-10 à j +21, car ils pourraient inhiber l'action du PRP [2,20,21]. Après la période algique, une rééducation excentrique sous-maximale, d'intensité progressive, associée à des étirements, permettra de «guider» la cicatrisation tendineuse [22]. Chez l'animal, une mobilisation active du tendon infiltré améliore effectivement cette cicatrisation [23] ; la rééducation excentrique, plus efficace que le travail concentrique, peut modifier la structure tendineuse [24]. Durant les 4 à 6 premières semaines post-infiltratives, le repos sportif nécessaire évite la survenue de nouvelles lésions intra-tendineuses; seule la rééducation, réalisée sous le seuil de la douleur, sera autorisée [25].

Lors de la reprise sportive progressive (réathlétisation), toute éventuelle technopathie sera corrigée et le tendon sera quelque peu «protégé » à l'aide d'orthèses «fonctionnelles » (bracelet patellaire) ou d'un strapping [26].

Nous avons observé, dans nos études, sur l'emploi de PRP pour traiter des tendinopathies patellaires proximales, que les patients plus jeunes répondaient plus efficacement au traitement, et ce, vraisemblablement en raison d'un meilleur potentiel de cicatrisation [25,27]. Ceux qui souffraient depuis moins de 10,5 mois évoluaient plus favorablement [27]. Les patients présentant initialement un meilleur capital quadricipital isocinétique en excentrique à $30^{\circ} / \mathrm{s}$ et en concentrique à $60 \%$ s progressaient plus favorablement [25]. Les patients présentant une réduction algique significative après 3 mois poursuivaient leur évolution favorable jusqu'à un an [27]. Le bilan à 3 mois évalue l'effet de l'infiltration de PRP et il permettrait de discuter l'indication d'une seconde infiltration de PRP en cas d'amélioration partielle de la symptomatologie tendineuse [27,28]. En effet, 2 infiltrations successives PRP (15 jours d'intervalle) ne semblent pas se justifier [29]. La chirurgie sera discutée en l'absence de toute amélioration [27].

\section{Conclusion}

Le PRP représente une thérapeutique récente et controversée des tendinopathies chroniques. Une standardisation de son emploi demeure nécessaire afin d'en améliorer les résultats. La première étape passe par la sélection des bonnes indications 
thérapeutiques et la correction des facteurs de risque des tendinopathies. Ensuite, l'optimisation de la technique d'obtention du PRP est primordiale tout comme le protocole d'injection. Enfin, une rééducation post-infiltration demeure absolument nécessaire.

\section{Déclaration d'intérêts}

Les auteurs déclarent ne pas avoir de conflits d'intérêts en relation avec cet article.

\section{Références}

[1] Kaux JF, Crielaard JM. Platelet-rich plasma application in the management of chronic tendinopathies. Acta Orthop Belg 2013;79(1):10-5.

[2] Smets F, Croisier JL, Forthomme B, Crielaard JM, Kaux JF. Applications cliniques du plasma riche en plaquettes (PRP) dans les lésions tendineuses: revue de la littérature. Sci Sport 2012;27:141-53.

[3] Dohan Ehrenfest DM, Rasmusson L, Albrektsson T. Classification of platelet concentrates: from pure platelet-rich plasma (P-PRP) to leucocyte- and platelet-rich fibrin (L-PRF). Trends Biotechnol 2009;27(3): $158-67$.

[4] Weibrich G, Kleis WK, Hitzler WE, Hafner G. Comparison of the platelet concentrate collection system with the plasma-rich-in-growth-factors kit to produce platelet-rich plasma: a technical report. Int J Oral Maxillofac Implants 2005;20:118-23.

[5] Lopez-Vidriero E, Goulding KA, Simon DA, Sanchez M, Johnson DH. The use of platelet-rich plasma in arthroscopy and sports medicine: optimizing the healing environment. Arthroscopy 2010;26(2):269-78.

[6] McCarrel TM, Minas T, Fortier LA. Optimization of leukocyte concentration in platelet-rich plasma for the treatment of tendinopathy. J Bone Joint Surg Am 2012;94:e143 [141-148].

[7] Boswell SG, Schnabel LV, Mohammed HO, Sundman EA, Minas T, Fortier LA. Increasing platelet concentrations in leukocyte-reduced platelet-rich plasma decrease collagen gene synthesis in tendons. Am J Sports Med 2014;42:42-9.

[8] Giusti I, D'Ascenzo S, Manco A, Di Stefano G, Di Francesco M, Rughetti A, et al. Platelet concentration in platelet-rich plasma affects tenocyte behavior in vitro. Biomed Res Int 2014:630870, http://dx.doi.org/10.1155/2014/630870 [Epub 2014 Jul 23].

[9] Moog R, Zeiler T, Heuft HG, Stephan B, Fischer EG, Kretschmer V, et al. Collection of WBC-reduced single-donor PLT concentrates with a new blood cell separator: results of a multicenter study. Transfusion 2003;43(8):1107-14.

[10] Pizza FX, McLoughlin TJ, McGregor SJ, Calomeni EP, Gunning WT. Neutrophils injure cultured skeletal myotubes. Am J Physiol Cell Physiol 2001;281(1):C335-41.

[11] Anitua E, Sanchez M, Prado R, Orive G. The type of platelet-rich plasma may influence the safety of the approach. Knee Surg Sports Traumatol Arthrosc 2014;22:1708-9.

[12] Dragoo JL, Braun HJ, Durham JL, Ridley BA, Odegaard JI, Luong R, et al. Comparison of the acute inflammatory response of two commercial platelet-rich plasma systems in healthy rabbit tendons. Am J Sports Med 2012;40:1274-81.
[13] Intravia J, Allen DA, Durant TJ, McCarthy MB, Russell R, Beitzel K, et al. In vitro evaluation of the anti-bacterial effect of two preparations of platelet rich plasma compared with cefazolin and whole blood. Muscles Ligaments Tendons J 2014;4:79-84.

[14] Kaux JF, Forthomme B, Le Goff C, Crielaard JM, Croisier JL. Current opinions on tendinopathy. J Sports Sci Med 2011;10:238-53.

[15] Chen SK, Lu CC, Chou PH, Guo LY, Wu WL. Patellar tendon ruptures in weight lifters after local steroid injections. Arch Orthop Trauma Surg 2009;129(3):369-72.

[16] Stinner DJ, Orr JD, Hsu JR. Fluoroquinolone-associated bilateral patellar tendon rupture: a case report and review of the literature. Mil Med 2010;175(6):457-9.

[17] Fichez O. Bonnes et mauvaises indications de l'injection de PRP dans les tendinopathies chroniques du sportif. In: Bard H, et al., editors. Le tendon et son environnement. 2013. p. 243-52 [Sauramps Médical].

[18] Loftus ML, Endo Y, Adler RS. Retrospective analysis of postinjection ultrasound imaging after platelet-rich plasma or autologous blood: observational review of anatomic distribution of injected material. AJR Am J Roentgenol 2012;199(4):W501-5.

[19] Engebretsen L, Steffen K, Alsousou J, Anitua E, Bachl N, Devilee R, et al. IOC consensus paper on the use of platelet-rich plasma in sports medicine. Br J Sports Med 2010;44(15):1072-81.

[20] Foster TE, Puskas BL, Mandelbaum BR, Gerhardt MB, Rodeo SA. Plateletrich plasma: from basic science to clinical applications. Am J Sports Med 2009;37(11):2259-72.

[21] Nguyen RT, Borg-Stein J, McInnis K. Applications of platelet-rich plasma in musculoskeletal and sports medicine: an evidence-based approach. PMR 2011;3(3):226-50.

[22] Kaux JF, Forthomme B, Namurois MH, Bauvir P, Defawe N, Delvaux F, et al. Standardized sub-maximal eccentric program after infiltration of platelet-rich plasma for jumper's knee. Muscles Ligaments Tendons J 2014;4(1):85-9.

[23] Virchenko O, Aspenberg P. How can one platelet injection after tendon injury lead to a stronger tendon after 4 weeks? Interplay between early regeneration and mechanical stimulation. Acta Orthop 2006;77(5):806-12.

[24] Kaux JF, Drion P, Libertiaux V, Colige A, Hoffmann A, Nusgens B, et al Eccentric training improves tendon biomechanical properties: a rat model. J Orthop Res 2013;31(1):119-24.

[25] Kaux JF, Forthomme B, Namurois MH, Bauvir P, Defawe N, Delvaux F, et al. Description of a standardized rehabilitation program based on submaximal eccentric following a platelet-rich plasma infiltration for jumper's knee. Muscles Ligaments Tendons J 2014;4(1):85-9

[26] Clifford AM, Harrington E. The effect of patellar taping on squat depth and the perception of pain in people with anterior knee pain. J Hum Kinet 2013;37:109-17.

[27] Kaux JF, Bruyère $\mathrm{O}$, Croisier JL, Forthomme B, Le Goff C, Crielaard JM. One-year follow-up of platelet-rich plasma infiltration to treat chronic upper patellar tendinopathies. Acta Orthop Belg [Sous presse].

[28] Dallaudière B, Meyer P, Hummel V, Perozziello A, Peuchant A, MoreauDurieux MH, et al. Efficacy of second intra-tendinous platelet-rich-plasma injection in case of incomplete response of the first injection: three-year follow up experience. Diagn Interv Imaging 2013;94(9):871-7.

[29] Kaux JF, Croisier JL, Forthomme B, Le Goff C, Buhler F, Savanier B et al. Using platelet-rich plasma to treat upper patellar tendinopathies: exploring the effect of a second closely-timed infiltrative administration. [Sous presse]. 